\title{
The Dynamics of a Japanese Reflexive Pronoun
}

\author{
Eric McCready \\ University of Texas at Austin \\ mccready@mail.utexas .edu
}

\begin{abstract}
This paper is concerned with the interpretation of the 'longdistance reflexive' zibun. I propose a new way to handle this item designed to account for intersentential binding facts. The account is couched within a version of dynamic semantics enriched with a notion of perspective. In the final part of the paper I show that this approach can be extended to account for intrasententially bound and indexical uses of zibun.
\end{abstract}

The present paper explores an approach to the 'long-distance reflexive' zibun that starts with different assumptions than most accounts to be found in the literature, which focus on the syntactic properties of this object.* No syntactic analysis has been able to capture all the phenomena; although each author has had valuable insights, the existence of intersententially bound zibun shows that a purely syntactic account cannot be possible. I will argue that the correct way of thinking about intersentential zibun is as an element that is dynamically bound by a salient object in the discourse context. This move allows us to not only analyze the phenomenon of intrasententially bound zibun that previous researchers have concentrated on, but also to analyze facts about intersentential binding and indexical uses of this object.

\section{Background on Zibun}

Zibun has been analyzed in the syntactic literature as polysemous, with realizations as an ordinary reflexive requiring a c-command relation (Kuroda, 1965), as an "empathetic logophor" which is sensitive to the 'camera angle' used in the discussion (Kuno, 1987), and as a de se reflexive which serves to self-ascribe a property to the speaker (Oshima 2002; see also the analysis of Chinese ziji of (Pan 1997)). One impetus behind this work is that on the assumptions of GB binding theory in the 1980s and early 1990s (cf. Chomsky (1981)), reflexive pronouns must be bound in their governing category, either a clause or a NP. This analysis turns out not to work for a large class of pronouns, however, the long-distance reflexives, discussed by various authors. Japanese zibun is one example of a long-distance reflexive; they are also found in Icelandic and other Scandinavian languages, Italian, Chinese, and Korean. The possibility of binding zibun from outside the clause is exhibited by (1):

\footnotetext{
* To appear in a volume in the Lecture Notes in Computer Science series, Springer Verlag. Thanks to the audience at LENLS for helpful comments and discussion, especially Rick Nouwen, Norihiro Ogata, and Katsuhiro Yabushita.
} 
(1) Taro-wa Ziro-ga zibun-wa atama-ga ii to itta to T-TOP Z-NOM self-NOM head-NOM good COMP say-PST COMP itta say-PST

'Taro ${ }_{i}$ said that Ziro $_{j}$ said that he ${ }_{i, j}$ is smart.'

Here Taro can bind zibun despite the fact that they appear in different clauses.

The most popular GB-style analyses of this problem involve covert movement at LF, where binding theory is sometimes taken to apply; a c-command relation between binder and bindee can be introduced by movement of one of these objects, allowing one to bind the other (Chomsky, 1981). These approaches successfully capture the general tendency of zibun to prefer binding by the subjects of clauses; this tendency has also played a role in analyses based on logophoricity. However, the proposed restriction is too strong; GB-based approaches incorrectly predict that only binding from subject position is possible; but zibun can be bound by objects within NPs or within adjunct clauses. Although there is a clear preference for binding by subjects, it is not difficult to find cases in which binding of zibun by elements in non-subject positions is possible, as in the following examples.

(2) Binding by antecedent in adjunct (Kameyama 1985):

Yamada-sensei-wa Taroo ni-totte zibun-no oya-no yoona

Prof. Yamada-ToP Taro for self-GEN parent-GEN existence

sonzai datta

COP-PST

'For Taro $_{i}$, Prof. Yamada was like his $_{i / * j}$ own parent.'

(3) Binding by NP-internal antecedent (Iida 1996):

Hanako-no ziman-wa zibun-no musuko da

Hanako-GEN pride-TOP self-GEN son COP

'Hanako $i$ 's pride is her ${ }_{i}$ son.'

Perhaps the most successful reanalysis to date that incorporates this other data has been that of Iida (1996), who uses a version of HPSG binding theory using notions of argument hierarchies and o-command to solve the problem (Pollard and Sag, 1994). The idea is essentially that an instance of zibun in a given grammatical role can be bound only by an argument in a higher position on the o-command hierarchy. Iida's theory pairs this notion with a set of pragmatic constraints, which together are very successful in accounting for the distribution of intrasententially bound zibun.

Intersentential binding of zibun is also possible in certain cases, as Iida notes, although her account doesn't extend to them due to its syntactic nature. The following examples are from Iida (1996); there she claims that the possibility of binding has to do with changes in perspective. For a coherent binding, one must maintain the perspective of the sentence in which the binder is introduced in the sentence that includes the instance of zibun to be bound: 
(4) Ziro-wa totemo okkotteimasu. Taro-ga zibun-no tomodati-ni [Ziro-ga Z-top very angry-is. T-nom self-gen friend-dat [Z-nom kaita] e-o misete-simatta kara desu drew] picture-acc show-perf-pst because cop.

'Ziro $i$ is very angry. It's because Taro ${ }_{j}$ showed his $_{i, j}$ friend a picture that Ziro drew.'

(5) Hanako-wa kinoo hidoku Taroo ni attattemasita. * Ziroo-ga H-top yesterday badly $\mathrm{T}$-dat bit-at Z-nom today kyoo zibun-ni au koto-ni natteimasu self-dat meet comp to is-planned

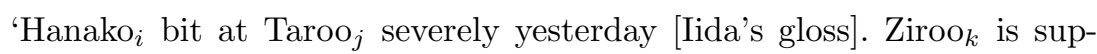
posed to see her ${ }_{i}$ today.'

(6) Hanako-wa kinoo hidoku T-ni atattemasita. Ziro-ga zibun-o H-top yesterday badly T-dat bit-at Z-nom self-acc mattaku musisita kara desu entirely ignored because cop 'Hanako $i$ bit at $\mathrm{T}_{j}$ severly yesterday. It is because Ziro ${ }_{k}$ ignored her ${ }_{i}$ completely.'

Iida comments that the reason for the coherence of these discourses is that the second sentence provides an explanation of the first, which allows the correct maintenance of perspective. Supporting this idea is the use of kara in the second sentences of the good discourses, which indicates the existence of a causal relation and the intention of the speaker to use the sentence as an explanation of what came before.

However, it seems that the situation of intersentential binding is more complicated than Iida suggests, in that a causal relation is not required for anaphora to be possible. Consider the following discourses ((8) is modeled on a Chinese example from Zhu (1997:89)):

(7) a. Taro-ga kore-o tabero to itta wake? Taro-NOM this-ACC eat-IMP C said actually 'Taro said to eat this?'

b. soo da. zibun-ga kirai-na kuse-ni.. yes COP. ZIBUN-NOM dislike even-though

'Yes. Even though he doesn't like it himself.'

(8) Taro-wa hontoo-ni fuun-na hito da. Saikin okusan-ga Taro-TOP really unlucky person COP. Recently wife-NOM kuruma-ni hikareta. kondo-wa zibun-ga kubi ni natta. car-DAT hit-PASS. next ZIBUN-NOM was-fired

'Taro is really an unlucky guy. Recently his wife got hit by a car. Now he got fired.'

No causal relations are to be found in the discourse in (8). Rather, it seems that the second and third sentence simply provide details about the proposition 
expressed by the first sentence. In the small dialogue in (7), the situation is even more clear: there cannot be a causal relation between the question and the answer given the semantic type of questions, which are standardly analyzed as sets of propositions. It is not clear how a set of propositions could itself be the cause of anything. ${ }^{1}$

Below I propose an account which is capable of providing a principled explanation for these cases. It is well known that discourses are not unstructured sequences of propositions, but have complex structures of their own (cf. Mann and Thompson 1986; Lascarides and Asher 1993; Webber et al. 2001; discourse segments (generally sentences or clauses) can be connected by various discourse relations. Recent work in Segmented Discourse Representation Theory (SDRT; Asher and Lascarides 2003) claims that discourse relations can be separated into two basic types, coordinating and subordinating relations. I claim that the possibility of using zibun intersententially crucially depends on the type of discourse relation that connects the sentences containing the (potential) antecedent and the instance of the reflexive: in particular, it must be a subordinating relation.

In SDRT, each discourse segment is treated as a speech act which introduces a label that marks its propositional content. Through a complex reasoning process involving nonmonotonic inference over discourse content, lexical information and world knowledge, binary discourse relations are inferred as holding between these labelled speech acts. The structure inferred is an acyclic graph which puts important constraints on anaphora: informally stated, for an anaphoric expression introduced in a given discourse segment $K$, only discourse referents introduced in segments which are connected to $K$ by some (sequence of) discourse relations are available. Importantly, once a segment is attached to a node $i$ in the discourse structure using a coordinating relation, nodes subordinate to $i$ are no longer available for attachment. This definition has important consequences for anaphora. Coordinating relations can be understood as relating two speech act labels on the same level of a hierarchical graph, while subordinating relations introduce dependent nodes. Thus, discourse referents introduced by a speech act $K$ connected to $K^{\prime}$ by a subordinating relation becomes unavailable for anaphoric expressions in a discourse segment $K^{\prime \prime}$ which is attached to $K^{\prime}$ by a coordinating relation.

Let us consider a concrete example.

(9) a. John had a wonderful evening last night. $\left(\pi_{1}\right)$

b. He had a great dinner. $\left(\pi_{2}\right)$

c. He ate salmon. $\left(\pi_{3}\right)$

d. He had a nice cheese. $\left(\pi_{4}\right)$

e. Then he won a dancing competition. $\left(\pi_{5}\right)$

f. ?It was a beautiful pink. $\left(\pi_{6}\right)$

\footnotetext{
${ }^{1}$ Note that I do not mean to claim that the speech act of asking a question cannot participate in causal relations, but that the semantic objects described by questions cannot be causal.
} 
In SDRT, Elaboration is a subordinating relation inferred when one segment gives more detail on the information provided by a preceding one: thus we infer Elaboration $\left(\pi_{1}, \pi_{2}\right)$, Elaboration $\left(\pi_{2}, \pi_{3}\right)$, Elaboration $\left(\pi_{2}, \pi_{4}\right)$, and

Elaboration $\left(\pi_{1}, \pi_{5}\right)$. The presence of the discourse marker then induces a monotonic inference of $N$ arration $\left(?, \pi_{5}\right)$; world and lexical knowledge allow resolution of ? to $\pi_{2}$. Narration is a coordinating relation, and so 'closes off' the possibility of attaching $\pi_{6}$ to $\pi_{3}$, as would be needed to resolve $i t$ to the referent introduced by salmon. The result is that the discourse is incoherent.

I wish to suggest in the present paper that the distinction between coordinating and subordinating relations is important not only for anaphoric accessibility in later segments, as is now quite standardly accepted, but also has consequences for the referent pool available for certain kinds of anaphoric expressions. The idea is that subordinating relations are able to maintain a referent pool, but coordinating relations 'empty' it. Zibun, I argue, is one instance of an expression sensitive to this distinction.

\section{Zibun dynamically}

One way to model the action of zibun is as an object which lacks inherent restrictions on its content; rather, a pool of referents exists that it can draw from that changes relative to what has been and what is being said. This pool of referents can be understood as a set of possible perspectives from which the situation described by a sentence or discourse can be viewed, where each perspective makes certain referents available for zibun in a way distinct from simple dynamic introduction of a discourse referent. Within the available referents, furthermore, one will be taken to be preferred to the others given the right circumstances. These circumstances include the use of inherently perspectival verbs or honorifics, and also facts about the world that enter into the inferencing mechanisms of the 'ordinary' language user.

I formalize this idea in the following way. taking standard DPL as a starting point (Groenendijk and Stokhof, 1991). I add a notion of a perspective and a mechanism for collecting antecedents for zibun; the set thus produced will be emptied with a change of perspective. I give the resulting logic the name 'RDPL'. ${ }^{2}$

Models for RDPL are defined just as in DPL, as are interpretation functions. Assignments look slightly different: we need a set of distinguished objects that can serve as antecedents for zibun, so instead of just assigning objects to variables, assignments in RDPL also have such a set associated with them. This set is notated $P_{n}$ and is updated with processing of each bit of logical form in tandem with the standard assignment.

\section{Definition 1. Assignments.}

$$
-f_{P_{n}}(x) \in D
$$

\footnotetext{
2 'Reflexive Dynamic Predicate Logic.'
} 
What is the content of the set $P_{n}$ ? That depends on where we are in a discourse. I assume that the only semantic object capable of introducing elements into $P_{n}$ is the existential quantifier. Thus the only clause of the DPL semantics that sees a serious change is for the existential quantifier. The rest have no effect on $P_{n}$. I use a DRT-style treatment of proper names, which often serve as antecedents for zibun, on which they are represented as existential quantifiers associated with a condition (e.g. john) which picks out a singleton set (cf. Kamp and Reyle 1993). Clauses for disjunction, implication, and universal quantification can be derived in the usual way.

\section{Definition 2. Semantics of RDPL Formulas.}

$$
\begin{aligned}
- & \llbracket R\left(x_{1}, \ldots, x_{n}\right) \rrbracket=\left\{\left\langle g_{P_{n}}, h_{P_{n+1}}\right\rangle \mid h=g \wedge\left\langle\llbracket t_{1} \rrbracket, \ldots, \llbracket t_{n} \rrbracket\right\rangle \in F(R) \wedge P_{n}=\right. \\
& \left.P_{n+1}\right\} \\
- & \llbracket \neg \varphi \rrbracket=\left\{\left\langle g_{P_{n}}, h_{P_{n+1}}\right\rangle \mid h=g \wedge \neg \exists k:\langle h, k\rangle \in \llbracket \varphi \rrbracket\right\} \\
- & \llbracket \varphi \wedge \psi \rrbracket=\left\{\left\langle g_{P_{n}}, h_{P_{n+1}}\right\rangle \mid \exists k:\langle g, k\rangle \in \llbracket \varphi \rrbracket \wedge\langle k, h\rangle \in \llbracket \psi \rrbracket\right\} \\
- & \llbracket \exists x \varphi \rrbracket=\left\{\left\langle g_{P_{n}}, h_{P_{n+1}}\right\rangle \mid \exists k: k[x] g \wedge\langle k, h\rangle \in \llbracket \varphi \rrbracket \wedge P_{n+1}=P_{n} \cup\{x\}\right\}
\end{aligned}
$$

Note that since the existential quantifier may introduce objects into $P_{n}$, the universal quantifier is predicted by the semantics of DPL to do the same, for its nuclear scope. This prediction is borne out, as shown by the donkey sentence in (10):

(10) subete-no otoko-wa tomodati-o zibun-no heya-ni yonda every man-TOP friend-ACC ZIBUN-GEN room-DAT invite-PST

'Every $\operatorname{man}_{i}$ invited a friend to his ${ }_{i}$ room.'

Now we move to the heart of the matter, the interpretation of zibun itself and the introduction of a dynamic notion of perspective. We have already done the groundwork by introducing the set $P$ and its update mechanism; it remains to show how it is used by zibun. This is basically very simple. Zibun is assumed to freely choose any member of $P_{n}$ as its referent; this is modelled by use of a choice function $h$. Which member is chosen will depend on various factors: world knowledge, grammatical elements, and what the interpreter perceives to be the intentions of the speaker; this last will be picked up on based on speech act used and so on discourse structure.

Perspectives are formalized in a simplistic way in this preliminary proposal, simply as a set of possible antecedents for zibun. Ultimately, of course, a perspective is more than this: ultimately, it should be considered as something more like an epistemic state, a set of propositions believed true by a perspective-holder, as well as a representation of the current state of a discourse. ${ }^{3}$ I also treat the notion of perspective change in a simplified manner. To really do the notion justice we must understand exactly how a perspective is changed, and this seems to depend rather heavily on all the factors mentioned in the preceding paragraph. Since I do not at present have a comprehensive theory of perspectives, I allow arbitrary

\footnotetext{
${ }^{3}$ See Asher (1992) for a treatment of perspectives in the context of a formal treatment
} of the semantics of progressive aspect. 
choice of points at which a perspective is changed, which I will model by use of a transition formula $X$, a 'blocker,' which affects output assignments by resetting the content of the antecedent set $P_{n}$ but has no effect on the truth-conditional content of the discourse. Such a formula is stipulated to appear at the conclusion of each single sentence, except when it is connected to the following sentence by a subordinating discourse relation, as shown by the examples of intersentential binding above (cf. Asher and Lascarides 2003).

\section{Definition 3. Semantics of Zibun and Perspective Changer}

$$
\begin{aligned}
& -P_{0}=\emptyset \\
& -\llbracket z i b u n \rrbracket=h\left(P_{n}\right) \\
& -\llbracket X \rrbracket=\left\{\left\langle g_{P_{n}}, h_{P_{n+1}}\right\rangle \mid g=h \wedge P_{n+1}=P_{n} \cap P_{0}\right\}
\end{aligned}
$$

Let's examine two simple examples to show how the system applies. We will use (6). The logical form of this discourse is shown in (11a), while (11b) shows the content of $P$ at each stage of update. Since $\pi$ and $\pi^{\prime}$ are connected by a subordinating relation, no blocking formulas are present, and so both Hanako $(x)$ and Taro $(y)$ are possible referents for zibun according to the present theory.

$$
\begin{aligned}
& \text { a. } \pi: \exists x[h(x)] ; \exists y[t(y)] ; \text { bite_at }(h, t) ; \pi^{\prime}: \exists z[z(z)] ; \text { ignore }(z, z \text { ibun }) ; \\
& \text { Explanation }\left(\pi^{\prime}, \pi\right) \\
& \text { b. }\{x\} ;\{x, y\} ;\{x, y\} ;\{x, y, z\} ;\{x, y, z\} ;\{x, y, z\}
\end{aligned}
$$

In the case of (5), however, the discourse relation is Narration, a coordinating relation; thus, blocking formulas appear after the content of each speech act, and intersentential binding is impossible, as shown in (12).

$$
\begin{aligned}
\text { a. } & \pi: \exists x[h(x)] ; \exists y[t(y)] ; \text { bite_at }(h, t) ; X ; \pi^{\prime}: \exists z[z(z)] ; \\
& \text { meet_today }(z, z i b u n) ; X ; \text { Narration }\left(\pi^{\prime}, \pi\right) \\
\text { b. } & \{x\} ;\{x, y\} ;\{x, y\} ; \emptyset ;\{z\} ;\{z\} ; \emptyset ; \emptyset
\end{aligned}
$$

It should also be noted here that, given the way the update mechanism is defined, intrasentential binding of zibun is assumed to take place via a different mechanism. It is not clear whether a unified account is desirable, given that somewhat different constraints seem to operate on intra- vs. intersentential zibun. The next section considers possible ways to extend the present account to the intrasentential case, should one decide that doing so is the right way to go.

\subsection{The intrasentential case}

Extension to intrasentential binding is not simple, for a number of reasons. First, it is well known that the referent selected by zibun depends, not only on pragmatic and discourse facts, but also on such hard-to-capture things as the degree of empathy shown by a particular utterance containing zibun with the individuals described in the sentence, as expressed by the particular lexical items chosen. For instance, certain complex verbs express a degree of empathy with the agent or patient of the described event. Second, the way the RDPL formalism is set up 
causes difficulties for compositional interpretation: given the left-to-right nature of dynamic binding, it will be necessary to force possible antecedents to precede zibun in logical form regardless of the surface order of the clauses. This factor will cause problems in case zibun appears in a clause-initial adjunct, for instance. Of course, it is possible to construct a working system (for instance by allowing each proper name to introduce an existentially quantified variable which is attached at the leftmost point of the formula introduced by the clause), but it is difficult to construct one that does not appear ad hoc. For this reason, the logic introduced in the present paper applies only to the intersentential case. In this section I confine myself to some discussion on how the first problem could be overcome, given a satisfactory solution for the second.

An attractive possibility is to utilize Iida's (1996) analysis, which crucially involves the HPSG o-command hierarchy (on grammatical roles). This can be done by annotating discourse referents (in $P$ ) with grammatical role information at the time of their introduction. Concretely, one might make use of the $\mathrm{f}$ (unctional)-structures of Lexical-Functional Grammar (Bresnan, 2001). In this theory, constituent structure maps to a functional structure in which grammatical roles are mapped to unique objects, as shown in (13):

(13) a. John likes Bill.

b. $\left[\begin{array}{ll}\text { PRED } & \text { 'LIKES }\langle\text { SUBJ, OBJ }\rangle ' \\ \text { SUBJ } & \text { 'John' } \\ \text { OBJ } & \text { 'Bill' }\end{array}\right]$

LFG f-structures contain all necessary information for discourse referent annotation. A function-argument pair of the form 〈SUBJ,'John' $\rangle$ can be defined as introducing an object of the form $j_{s u b j}$ into $P_{n} \cdot{ }^{4}$ Lexical entries for words/morphemes such as morau/kureru which indicate 'empathy' with one particular element then bias the interpretation of zibun toward the correct argument by selecting for an element with a particular subscript. If such an analysis is adopted, it becomes clear that we need not lose empirical coverage by switching to a dynamic interpretation of zibun, but rather the opposite. By adopting a dynamic account, we become able in principle to provide a unified account of intersentential and intrasentential binding of zibun.

Oshima (2002) and others note that zibun is associated with de se readings, readings of attitude-ascribing sentences in which a speaker self-ascribes an attitude. To see what this reading consists of, consider the following example due to Kaplan (1989):

(14) John believes that his pants are on fire.

a. John believes that John's pants are on fire.

b. John believes that his own pants are on fire.

(14) is ambiguous between the two readings in (14a) and (14b). Consider a situation in which John is looking at a mirror, which reflects an individual with

\footnotetext{
${ }^{4}$ The same trick would work in HPSG, of course.
} 
his pants on fire. Imagine now that John does not realize that he is looking at his own reflection - that is, he thinks that the individual with burning pants is not him, but someone else. This reading is described by (14a). If John realizes that he is looking at himself, the sentence would have the reading in (14b). Assuming that John is completely amoral, only the second reading is one that would prompt him to try to put the fire out, for only this reading is 'self-directed' in the way that would cause him to act. The reading described by (14b) is the de se reading.

It turns out that when zibun is used the second reading is preferred; that is, zibun forces a de se reading. Within the current dynamic semantic setting, the different readings are distinguished by use of an anchoring function from discourse referents to objects: this function can be partial, to allow for the possibility that a speaker's mental representation of the discourse involves an object that actually doesn't exist (see Kamp et al. 2003 for discussion). De se readings are obtained by mapping the discourse referent representing the subject of the embedded proposition to the speaker by the anchoring function (Dekker, 2000). To handle the Japanese facts, anchoring functions can be further constrained so that they always map zibun to the attitude holder (in the case of potentially de se attitudes). Space considerations preclude spelling this analysis out in the present paper.

\subsection{The indexical case}

Zibun also has an indexical use: it can refer to the speaker of the utterance in which it appears, or to the interlocutor in certain special circumstances. In standard (Tokyo) Japanese and also in various dialects, an interpretation of zibun as an indexical referring to the speaker is available. Apparently this use comes from military jargon of the early 20th century, when members of the Japanese armed forces were required to refer to themselves as zibun when speaking to superiors (Martin, 1975); somehow this usage spread into everyday speech, and is now very common. As far as I can tell, this usage is available quite generally, although it is restricted to relatively colloquial speech. Here is an instance:

(15) zibun-wa hara het-teiru kedo.

self-TOP stomach empty-PROG though

'I'm hungry now anyway.'

The remaining indexical use exists only in certain dialects of Japanese. It's primarily associated with the dialect of the Kansai region (comprising, generally speaking, the region around Osaka and Kyoto), though it also appears in other Western dialects in Kyushu and the Chugoku region. Zibun in these dialects can also refer to the interlocutor; that is, it can also mean 'you,' as in (16):

(16) zibun-no suru koto-wa zenbu tadasii to omo-tteiru kara self-GEN do thing-TOP all correct COMP think-PROG because yaro probably 
'(That's) because you think everything you (I) do is right (isn't it).'

Note that zibun here can refer either to the speaker or to the hearer, though the second person interpretation is probably more natural. This second person use of reflexive pronouns turns out to be quite common cross-linguistically: similar facts can be found in Korean, Norwegian, and Kannada (Jeffrey Lidz, p.c.), and probably other languages as well.

We can account for indexical uses of zibun through a simple modification of the RDPL system described above. Above we assumed that the base set for update of possible zibun-antecedents, $P_{0}$, was the empty set. We need only assume that the base set is actually not empty to account for the possibility of zibun denoting the speaker, as follows:

$$
\text { - } P_{0}=\{s\}
$$

For dialects of Japanese which permit reference to the interlocutor, we can add another element to this set $(i)$. One might object to this move on the assumption that the second person use is simply a different lexical item than the first, for example with a different tone; if correct, this would motivate assuming the existence of two distinct base sets $P_{0 s}$ and $P_{0 i}$. However, this is not the case, as McCready (2003) showed through phonetic experiments involving production data. Further, no phonetic difference is perceivable by interpreters (McCready, 2003), which also suggests that only one lexical item is involved.

Note that this semantics predicts that use of zibun is universally available to denote speaker or hearer. In fact, this prediction is probably not completely correct. First person indexical uses of zibun within the complements of attitude verbs seem to require focus-like intonational prominence. Also, the distribution of second person indexical zibun seems to be even more restricted in that it is felicitous primarily in the context of certain speech acts. I will not consider these complications further in the present paper.

\section{Summary}

In this paper, I proposed a new account of the Japanese long-distance reflexive zibun on which it is analyzed as a special sort of dynamically bound object associated with a set of potential binders. Each member of this set is taken to correspond to an individual from whose perspective the situation described by a sentence (or discourse) is viewed. Although the account is designed to handle cases of intersentential binding of zibun, I showed how it can be extended to cases of intrasentential binding and even indexical uses of zibun. 


\section{Bibliography}

Asher, Nicholas. 1992. A default, truth-conditional semantics for the progressive. Linguistics and Philosophy 15:463-508.

Asher, Nicholas and Alex Lascarides. 2003. Logics of Conversation. Cambridge University Press.

Bresnan, Joan. 2001. Lexical-Functional Syntax. Blackwell.

Chomsky, Noam. 1981. Lectures on Government and Binding. Dordrecht: Foris.

Dekker, Paul. 2000. Coreference and representationalism. In K. von Heusinger and U. Egli, eds., Reference and Anaphoric Relations. Dordrecht: Kluwer.

Groenendijk, Jeroen and Martin Stokhof. 1991. Dynamic predicate logic. Linguistics and Philosophy 14:39-100.

Iida, Masayo. 1996. Context and Binding in Japanese. Stanford: CSLI. Ph.D Thesis, Stanford University, 1992.

Kameyama, Megumi. 1985. Zero Anaphora: the Case of Japanese. Ph.D. thesis, Stanford University.

Kamp, Hans and Uwe Reyle. 1993. From Discourse to Logic. Dordrecht, Reidel: Kluwer.

Kamp, Hans, Josef van Genabith, and Uwe Reyle. 2003. Discourse representation theory. Draft of article to appear in Handbook of Philosophical Logic.

Kaplan, David. 1989. Demonstratives. In J. Almog, J. Perry, and H. Wettstein, eds., Themes from Kaplan. Oxford University Press. Manuscript version from 1977.

Kuno, Susumu. 1987. Functional Syntax: Anaphora, Discourse, and Empathy. University of Chicago Press.

Kuroda, Sige-Yuki. 1965. Generative Grammatical Studies in the Japanese Language. Ph.D. thesis, MIT.

Lascarides, Alex and Nicholas Asher. 1993. Temporal interpretation, discourse relations and commonsense entailment. Linguistics and Philosophy 16:437493.

Mann, William and Sandra Thompson. 1986. Relational propositions in discourse. Discourse Processes 9:57-90.

Martin, Samuel. 1975. A Reference Grammar of Japanese. New Haven: Yale University Press.

McCready, Eric. 2003. Prosodic contour and indexical interpretation in Osaka Japanese. unpublished ms.

Oshima, David Y. 2002. Logophoricity, empathy, and de se interpretation. Revised version of Tokyo University Masters' thesis.

Pollard, Carl and Ivan Sag. 1994. Head-Driven Phrase Structure Grammar. CSLI and University of Chicago Press.

Webber, Bonnie, Matthew Stone, Aravind Joshi, and Alastair Knott. 2001. Anaphora and discourse semantics. To appear in Computational Linguistics. 